\title{
Regulation of Intracellular Free Arachidonic Acid in Aplysia Nervous System
}

\author{
Robert O. Carlson* and Irwin B. Levitan \\ Graduate Department of Biochemistry, Brandeis University, Waltham, Massachusetts 02254
}

\begin{abstract}
Summary. We have studied the regulation of arachidonic acid (AA) uptake, metabolism, and release in Aplysia nervous system. Following uptake of $\left[{ }^{3} \mathrm{H}\right] \mathrm{AA}$, the distribution of radioactivity in intracellular and extracellular lipid pools was measured as a function of time in the presence or absence of exogenous AA. The greatest amount of AA was esterified into phosphatidylinositol (relative to pool size). We found that the intracellular free AA pool underwent rapid turnover, and that radioactive free $A A$ and eicosanoids were released at a rapid rate into the extracellular medium, both in the presence and absence of exogenous AA. Most of the released radioactivity originated from phosphatidylinositol.

Two pharmacological agents were found to modulate AA metabolism in Aplysia ganglia. The phorbol ester, 12-O-tetradecanoylphorbol 13-acetate, stimulated liberation of AA from phosphatidylinositol and phosphatidylcholine. This resulted in an increase in free internal and secreted AA, an increase in conversion of AA to eicosanoids, and an increase in esterification of AA into triacylglycerol. The half maximal dose for TPA-stimulated AA turnover was $15 \mathrm{nM}$, and the stimulation was dependent on the presence of extracellular calcium. 4-bromophenacylbromide inhibited the redistribution of radioactivity from phospholipid into triacylglycerol, indicating BPB was acting as a phospholipase inhibitor in Aplysia as it does in other systems. These pharmacological agents, in addition to providing information about the regulation of AA metabolism and release, are useful tools for investigating the physiological function of the rapid turnover of AA in Aplysia nervous system.
\end{abstract}

Key Words arachidonic acid $\cdot$ eicosanoids $\cdot$ Aplysia $\cdot$ phorbol ester $\cdot$ protein kinase C

\section{Introduction}

Arachidonic acid and its metabolites, or eicosanoids, have been implicated in the regulation of a wide variety of biological processes. Some of the best described physiological roles for eicosanoids involve pathological phenomena, such as tissue injury or immune responses $[13,34,47,54,72]$. The

* Present address: The University of Michigan, Neuroscience Building, 1103 East Huron Rd., Ann Arbor, MI 48109. molecular mechanisms that underlie eicosanoid activity in these phenomena are not well defined. However, $\mathrm{AA}^{1}$ and eicosanoids appear to play a role in the regulation of secretory processes in many tissues. In pancreatic islet cells, eicosanoids have been shown to mediate glucose-induced insulin secretion $[43,57,70,74]$. In the pituitary and hypothalamus, eicosanoids regulate secretion of many peptide hormones $[2,11,32,45,48,50,52]$. In certain identified Aplysia neurons, eicosanoids have been implicated in the signal transduction mechanism for the peptide hormone FMFRamide, which activates a potassium current to cause a decrease in transmitter release $[55,56]$. This was the first indication that eicosanoids may regulate secretion through modulation of cellular electrical activity.

The concentration of intracellular free $\mathrm{AA}$ is kept very low in cells and has been shown to be rate limiting for eicosanoid production [30]. Understanding the regulation of internal free AA, therefore, is crucial for the study of any physiological process involving AA and its metabolites. In this paper, we characterize the regulation of intracellular free AA in Aplysia nervous system. Our interest in Aplysia neural AA metabolism derives from the need for information and tools to help probe for possible AA-mediated modulation of neuronal electrical activity. In the accompanying paper [10], we exploit the information obtained from the biochemical approaches described here, to investigate the relationship between AA metabolism and electrical activity in identified Aplysia neurons.

${ }^{1}$ Abbreviations: AA, arachidonic acid; $\mathrm{AA}_{\mathrm{ext}}$, extracellular free $\mathrm{AA} ; \mathrm{AA}_{\text {int }}$, intracellular free AA; $\mathrm{AA}-\mathrm{CoA}$, arachidonyl coenzyme A; PL, phospholipid; PC, phosphatidylcholine; PI, phosphatidylinositol; PS, phosphatidylserine; PE, phosphatidylethanolamine; NL, neutral lipid; TG, triacylglycerol; TPA, 12-O-tetradecanoylphorbol 13-acetate; BPB, 4-bromophenacylbromide; PLA $A_{2}$, phospholipase $A_{2} ;$ PLC, phospholipase C; TLC, thin-layer chromatography. 


\section{Materials and Methods}

\section{ANimals AND REAGENTS}

Adult Aplysia californica (100 to $250 \mathrm{~g}$ ) were supplied by Alacrity Marine Biological Services (Redondo Beach, CA). To anesthetize prior to dissection, Aplysia were injected with up to $100 \mathrm{ml}$ of $0.4 \mathrm{M} \mathrm{MgCl}_{2}$. Ganglia were removed and immediately placed in artificial seawater (ASW, containing in mM: $460 \mathrm{NaCl}, 55$ $\mathrm{MgCl}_{2}, 11 \mathrm{CaCl}_{2}, 10 \mathrm{KCl}, 10 \mathrm{NaHEPES}, \mathrm{pH} 7.3$ ) with $1 \%$ (wt/ vol) glucose.

All reagents were of highest grade available. Nonradioactive AA and eicosanoids were purchased from Cayman Chemicals (Ann Arbor, MI). Lipid standards for thin-layer chromatography were from Avanti Polar Lipids (Birmingham, AL) or Sigma. Organic solvents and inorganic salts were from Fisher. $\left[{ }^{3} \mathrm{H}\right]$ arachidonic acid (sp act: $191-240 \mathrm{Ci} / \mathrm{mmol}$ ) was from New England Nuclear (Boston, MA). We found this $\left[{ }^{3} \mathrm{H}\right] \mathrm{AA}$ to be consistently at least $99 \%$ pure as determined by TLC. All lipids used in this study were stored under nitrogen at -20 or $-70^{\circ} \mathrm{C}$.

\section{LABELING WITH [3 $\left.{ }^{3}\right]$ AA AND RELEASE INCUBATIONS}

Pleural and pedal ganglia were used exclusively for results reported in this paper. From each Aplysia, the pleural and pedal pairs were separated with the right and left pleuropedal connectives intact. For simplicity, we refer to this connected pleural and pedal ganglion pair as an individual "pleuropedal" ganglion.

Ganglia were incubated individually for $2-3 \mathrm{hr}$ in ASW containing $\left[{ }^{3} \mathrm{H}\right] \mathrm{AA}$, and $0.05-0.1 \%$ (wt/vol) BSA (fatty acid free, from Sigma or Boehringer Mannheim Biochemicals). Aliquots of $\left[{ }^{3} \mathrm{H}\right] \mathrm{AA}$ were dried under nitrogen, and resolubilized in ethanol prior to solubilization in ASW. The final concentration of ethanol never exceeded $0.5 \%$ (vol/vol). To terminate labeling and remove $\left[{ }^{3} \mathrm{H}\right] \mathrm{AA}$ not sequestered intracellularly, ganglia were transferred through two to three changes of fresh ASW without AA.

To assay for release of $\mathrm{AA}$ and eicosanoids into the extracellular medium, and to follow distribution of radioactivity as a function of time and different incubation conditions, a labeled pleuropedal ganglion was placed in $1.5 \mathrm{ml}$ of $\mathrm{ASW}$ (with $0.1 \%$ BSA) kept in constant circulation. This was called "release incubation." During the release incubation, the medium was removed and replaced with fresh medium every $30 \mathrm{~min}$. The medium removed was kept on dry ice for later extraction and analysis of radioactivity in specific lipid pools.

This release incubation procedure was used to test the effects of various treatments on distribution of radioactivity in cellular and released lipids. Stock solutions of BPB (Sigma) were made up fresh in DMSO before experiments. Stock solutions of TPA (Sigma) were stored in single-use aliquots at $-20^{\circ} \mathrm{C}$. The final concentration of carrier DMSO in ASW was usually less than $0.1 \%(\mathrm{vol} / \mathrm{vol})$, and a matching concentration of DMSO was included in control solutions.

\section{Extraction AND ANALYSIS OF LipIDS}

\section{Extraction}

To analyze released radioactivity in more detail, the extracellular medium was extracted for lipid by acidification to $\mathrm{pH} 3$ with formic acid, followed by extraction with three volumes of ethyl acetate, two or three times. A modified version of the Folch method [23] was used to extract lipid from ganglia. Ganglia were crushed by hand in a Teflon and glass homogenizer containing $0.9 \mathrm{ml}$ of chloroform $\left(\mathrm{CHCl}_{3}\right)$ : methanol $(\mathrm{MeOH}): \mathrm{HCl}(\mathrm{vol} / \mathrm{vol} /$ vol, $1: 2: 0.02$ ), and $0.2 \mathrm{ml} \mathrm{ASW}$. This solution was removed, and replaced for a second extraction of the remaining tissue. These two extracts were pooled with $0.4 \mathrm{ml} \mathrm{CHCl}_{3}$ and $0.4 \mathrm{ml} \mathrm{H}_{2} \mathrm{O}$. After vortexing, the samples were centrifuged to separate the phases. The lower, organic solvent phase, or lipid extract, usually contained $75-80 \%$ of the radioactivity originally taken up by the ganglion. (In separate experiments, $\left[{ }^{3} \mathrm{H}\right] \mathrm{AA}$ was found to partition completely into the organic phase under these conditions.)

\section{Thin-Layer Chromatography}

Extracellular medium or ganglionic lipid extracts were dried in vacuo using a Savant Speed-Vac. Following removal of solvent, samples were immediately resolubilized in 10-20 $\mu \mathrm{l}$ $\mathrm{CHCl}_{3}: \mathrm{MeOH}$ (vol/vol 2:1), containing the anti-oxidant butylated hydroxytoluene $(100 \mathrm{mg} / \mathrm{liter})$, and stored under nitrogen. Resolubilized samples were applied in bands to pre-coated silica gel-60 thin layer plates (EM Science). For measuring changes in intracellular free AA, samples were mixed with $0.1 \mu \mathrm{g}$ nonradioactive AA (for visualization of the AA band with iodine vapor) and were developed in System I:petroleum ether: diethyl ether: acetic acid (vol/vol/vol, $80: 20: 1$ ) [46]. For separation of phospholipid, eicosanoids, and neutral lipid, a modification of the one-dimensional, double development system of Heape et al. [25] was used. Phospholipids were resolved with System II : methyl acetate : 1 -propanol : $\mathrm{CHCl}_{3}: \mathrm{MeOH}: 0.25 \%$ (wt/vol) $\mathrm{KCl}(\mathrm{vol} / \mathrm{vol} / \mathrm{vol} / \mathrm{vol} / \mathrm{vol}, 25: 25: 28: 10: 7)$. The plates were air-dried and then redeveloped with System I in the same direction to separate eicosanoids and neutral lipids (phospholipids are immobile in System I). $R_{f}$ values were variable (values from a representative run are given in parentheses in the following sequence), but the mobility sequence was invariant: PC $(0.05)<$ $\mathrm{PS}(0.11)<\mathrm{PI}(0.15)<\mathrm{PE}(0.28)<$ eicosanoids \{prostaglandin $\mathrm{F}_{2 \alpha}(0.5)<$ prostaglandin $\mathrm{E}_{2}(0.56)<5$-hydroxyeicosatetraenoic acid (5-HETE) $(0.66)<$ 15-HETE $(0.70)\}<$ AA $(0.73)<$ diooleoylglycerol $=$ triarachidonylglycerol $(0.76)<$ trioleoylglycerol $(0.94)$.

To visualize major phospholipids and neutral lipids, plates were exposed briefly to iodine vapor. For autoradiographic detection of lipids present in small quantities, plates were sprayed with $\mathrm{En}^{3}$ hance (New England Nuclear), wrapped in plastic wrap, and used to expose Kodak X-ray omat-R-film with aid of enhancing screens. Iodine-visualized or autoradiographic bands were used as guides for scraping plates to assay radioactivity present in individual lipid pools. Plate scrapings were suspended in $3 \mathrm{ml}$ of Beckman Ready-Solv $\mathrm{HP} / \mathrm{b}$ scintillation fluid for liquid scintillation counting in a Beckman LS6800 scintillation counter.

\section{Lipid Phosphate}

Lipid phosphate was assayed by first digesting lipid extracts or scrapings from thin-layer plates in $0.25 \mathrm{ml} 10 \mathrm{~N} \mathrm{H}_{2} \mathrm{SO}_{4}$ for $3 \mathrm{hr}$ at $160-180^{\circ} \mathrm{C}$ in acid washed tubes, as described by Keenan et al [33]. The subsequent steps were modified after Sanui [63]. After cooling, $1.8 \mathrm{ml}$ water, $3.0 \mathrm{ml}$ butyl acetate, and $0.9 \mathrm{ml} 2.25 \%$ ammonium molybdate were added sequentially, and the tubes 
were capped and vortexed for $30 \mathrm{sec}$. Absorbance of the upper: butyl acetate phase was measured at $311 \mathrm{~nm}$. Standard curves were generated from samples containing $25-100 \mathrm{nmol}$ inorganic phosphate.

\section{STATISTICS}

Unless otherwise stated, error bars in graphs represent the standard deviation of independent measurements from at least three ganglia. Where indicated in parentheses, the number of observations refer to the number of individual ganglia used for a particular experiment. Student's $t$ test was used to test for significant differences under different experimental conditions.

\section{Results}

\section{AA Metabolism in Aplysia Nervous System}

\section{Uptake}

Figure 1 depicts a simplified mechanism of AA metabolism derived from studies of fatty acid uptake $[15,16,67]$ and metabolism [61]. AA initially enters cells by nonenzymic partitioning into membrane lipid (pathway labeled $I$ in Fig. 1). Acyl-CoA synthetases convert AA in the membrane to arachidonyl-coenzyme A esters (AA-CoA), the activated form of AA required for esterification into lipid (II). Acyl-CoA transferases catalyze condensation of AA-CoA with lysophospholipid to produce phospholipid $(I V)$, or with diacylglycerol to produce triacylglycerol $(I I I)$. Once in storage, hydrolysis of AA from phospholipid is the primary source of internal free AA $(V)$. Several classes of enzymes, including cyclooxygenase, lipoxygenases, and epoxygenases, catalyze conversion of internal free AA to eicosanoids $(V I I)$.

To characterize the details of AA metabolism specific to Aplysia nervous system, we studied uptake and distribution of trace exogenous [ $\left.{ }^{3} \mathrm{H}\right] \mathrm{AA}$ in Aplysia pleuropedal ganglia. Although pleuropedal ganglia were used exclusively for the experiments reported here, we have obtained similar results in other Aplysia ganglia.

The Table lists the distribution of radioactivity in major lipid pools from ganglia labeled under similar conditions. In this and other experiments that involved labeling, total uptake of radioactivity was used to normalize for differences in amount of ganglionic tissue. We routinely express the content of radioactivity in AA-containing pools as "percent of total $\left[{ }^{3} \mathrm{H}\right] \mathrm{AA}$ uptake." For the normal labeling protocol, $\sim 5 \times 10^{6} \mathrm{dpm}$ were taken up into a single pleuropedal ganglion. Less than $1 \%$ of the total AA taken up $(\sim 50,000 \mathrm{dpm})$ was found as internal, free,

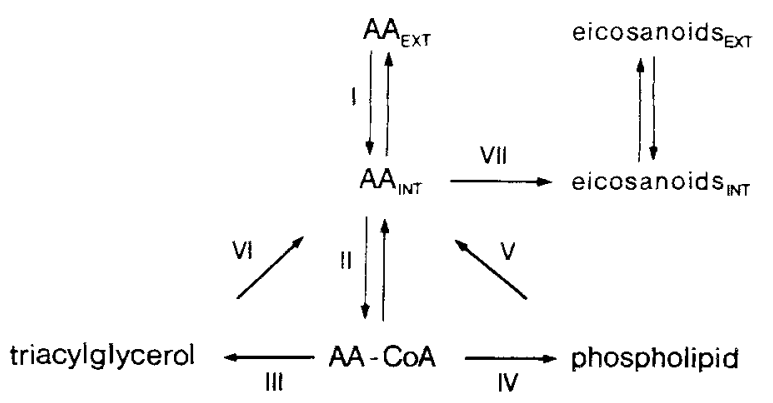

Fig. 1. AA metabolism in animal cells. Pathways for uptake of AA into and liberation from cellular glycerolipids and metabolism of AA to eicosanoids. Roman numeral-labeled pathways are catalyzed by the following activities: $(I)$ nonenzymic partitioning into membrane; (II) acyl-CoA synthetase; (III) diglyceride acylCoA transferase; $(I V)$ phospholipid acyl-CoA transferase; $(V)$ phospholipase(s); (VI) triacylglycerol lipase; (VII) several enzymes, including cyclooxygenase, lipoxygenases, and epoxygenases. This diagram does not include de novo pathways for incorporation of AA into lipids. Also, in the presence of an alternative energy source (e.g., glucose), $\beta$-oxidation is a minor pathway

unmetabolized AA. The majority of the AA taken up was esterified into either phospholipid or neutral lipid. Production of eicosanoids was detected by the appearance of bands of radioactivity which migrated in thin-layer chromatography with several authentic eicosanoids. This putative eicosanoid radioactivity was only detectable in the extracellular medium (autoradiographs of thin-layer chromatographs of intracellular lipids never contained distinct bands of radioactivity in the region of eicosanoid mobility), as could be predicted from the general observation that cells do not store eicosanoids [30]. These bands of putative eicosanoids were not due to autooxidation of AA. Six hours of incubation of $\left[{ }^{3} \mathrm{H}\right] \mathrm{AA}$ alone in ASW at room temp. did not produce bands of radioactivity which ran in the defined region of eicosanoid mobility.

The Table also lists the relative prevalence of individual phospholipids as measured by lipid phosphate content. Over half of the measured lipid phosphate was found in PC. Most of the remaining phosphate was found in PI, PS, and PE (in order of measured content). The ratio of AA uptake to lipid phosphate provides a measure of the amount of AA taken up relative to the phospholipid pool size. Based upon this ratio, two to three times as much radioactivity was esterified into $\mathrm{PI}$, relative to the PI pool size, as compared to other phospholipids.

AA uptake into ganglionic lipids was measured as a function of time. The partitioning of AA into specific pools was measured in 0.6 and $50 \mu \mathrm{M}$ AA. The percentage of AA found free or esterified into phospholipid or neutral lipid did not vary from 30 
Table. Average distribution of AA label and comparison to phospholipid pool size

\begin{tabular}{|c|c|c|c|}
\hline Lipid & $\%$ of uptake & $\%$ of lipid phosphate & $\begin{array}{l}\text { Uptake/ } \\
\text { phosphate }\end{array}$ \\
\hline \multicolumn{4}{|l|}{ Intracellular } \\
\hline Phosphatidylcholine & $27.1 \pm 2.9$ & $55.7 \pm 2.2$ & 0.49 \\
\hline Phosphatidylserine & $6.4 \pm 1.9$ & $12.5 \pm 1.8$ & 0.51 \\
\hline Phosphatidylinositol & $17.5 \pm 5.6$ & $18.6 \pm 1.6$ & 0.95 \\
\hline Phosphatidylethanolamine & $2.5 \pm 1.0$ & $8.6 \pm 0.6$ & 0.29 \\
\hline Triacylglycerol & $27.6 \pm 4.3$ & - & - \\
\hline Arachidonic acid & $0.6 \pm 0.3$ & - & - \\
\hline \multicolumn{4}{|l|}{ Extracellular } \\
\hline Arachidonic acid & $0.6 \pm 0.2$ & - & - \\
\hline Eicosanoids & $1.1 \pm 0.4$ & - & - \\
\hline
\end{tabular}

Data on distribution of AA label, expressed as percent of total $\left[{ }^{3} \mathrm{H}\right] \mathrm{AA}$ taken up, were averaged from experiments which involved extraction and analysis of lipid from ganglia labeled under similar conditions $(n=43)$. Label content of major cellular lipids are listed, representing $82 \%$ of the total $\left[{ }^{3} \mathrm{H}\right] \mathrm{AA}$ taken up. The remaining label was primarily in unidentified neutral lipids. The labeling incubations were all for $3 \mathrm{hr}$, ranging from $100-250 \mu \mathrm{l}$ of 100-270 nM [ $\left.{ }^{3} \mathrm{H}\right] \mathrm{AA}$ (sp act 190-240 Ci/mmol). In all experiments, ganglia were washed free of unsequestered label and placed in release incubation conditions (described in Materials and Methods). The release incubations were generally for $1 \mathrm{hr}$, though several were for 2 or $6 \mathrm{hr}$. (Even after $6 \mathrm{hr}$, release was a relatively small percentage of the total uptake; therefore, the difference in duration of release incubation did not appreciably alter the label distribution). The data for extracellular AA and eicosanoids are solely from $1 \mathrm{hr}$ release incubations $(n=24)$. Lipid phosphate, expressed as percent of total ganglionic lipid phosphate, was determined for individual phospholipids as described in Materials and Methods. The ratio of percent of $\left[{ }^{3} \mathrm{H}\right]$ AA taken up to percent of lipid phosphate provides an estimate of the amount of [ $\left.{ }^{3} \mathrm{H}\right] \mathrm{AA}$ taken up relative to the amount of the individual phospholipid.

min to $4 \mathrm{hr}$ of incubation in $0.6 \mu \mathrm{M}$. Except for an increase in radioactivity in triacylglycerol and a concomitant decrease in radioactivity in an unidentified neutral lipid which ran at the solvent front, this lack of time dependence for the distribution of radioactivity was observed for all other lipids extracted from ganglia incubated $10 \mathrm{~min}$ to $2 \mathrm{hr}$ in 50 $\mu \mathrm{M}$ AA.

We also assayed uptake of AA as a function of exogenous AA concentration. Esterification of AA into phospholipids began to approach saturation above $0.6 \mu \mathrm{M}$ exogenous AA (depicted in Fig. $2 \mathrm{~A}$ for PC, PI, and PS from ganglia incubated in $60 \mathrm{nM}$ to $25 \mu \mathrm{M}$ AA). Esterification into triacylglycerol continued to increase up to $25 \mu \mathrm{M}$ AA (Fig. $2 B$ ). This lack of saturation is in agreement with the nearly unlimited capacity of cells in culture for fatty acid uptake into triacylglycerol, which accumulates as liposomes in the cytoplasm of cells to the point of rupture [61]. Triacylglycerol was the main neutral lipid for AA storage. In $60 \mathrm{~nm}$ AA, $74 \%$ of the radioactivity found in neutral lipid was in triacylglycerol, increasing to $95 \%$ in $25 \mu \mathrm{M} \mathrm{AA}$. At the lowest concentrations tested (60-200 nM), uptake into phospholipid was two- to threefold greater than uptake into triacylglycerol; conversely, in $50 \mu \mathrm{M} \mathrm{AA}$, over five times as much AA was esterified into triacylglycerol. Accumulation of AA in the internal free AA pool did not saturate in up to $25 \mu \mathrm{M}$ AA (Fig. $2 C$ ). Therefore, at higher exogenous AA concentrations, flux into the free AA pool likely is coupled to the uptake from this pool into triacylglycerol.

\section{Release into Extracellular Medium}

Radioactivity was released from labeled ganglia into release incubation medium (ASW with $1 \%(\mathrm{wt} / \mathrm{vol})$ glucose and $0.1 \%(\mathrm{wt} / \mathrm{vol})$ fatty acid free BSA) at a constant rate. The presence of BSA was not required to observe release of radioactivity, but adding BSA at $1 \mathrm{mg} / \mathrm{ml}$ increased basal release about twofold. BSA was routinely included to act as a trap for lipids released from ganglia. $83 \pm 8 \%(n=35)$ of released radioactivity was contained in free $\mathrm{AA}$ (29\%) and eicosanoids (54\%); the remaining radioactivity ran in the region of neutral lipid mobility. This distribution of released radioactivity did not vary with time.

Figure 3 shows a comparison of radioactivity in free AA released into the extracellular medium with radioactivity in inträcellular free $\mathrm{AA}$ as a function of time. Internal free AA was defined as free AA radioactivity present in the extract of cellular lipids which was not removed previously by multiple washes of an intact ganglion with fresh medium. Radioactivity in released free AA increased steadily with time of incubation, while the amount of internal free AA radioactivity remained constant. There is no precedence for extracellular release of free AA from cellular lipid without the liberated AA first 


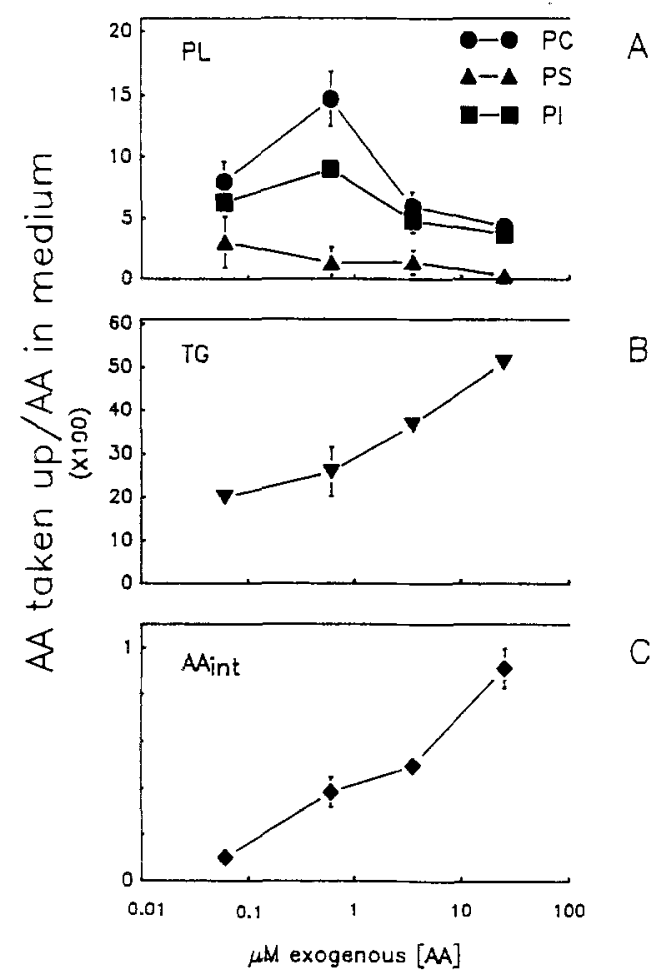

Fig. 2. AA uptake into intracellular pools. Ganglia were incubated individually for $2 \mathrm{hr}$ in different concentrations (60 nM-25 $\mu \mathrm{M}$ ) of exogenous AA (sp act: $15 \mathrm{Ci} / \mathrm{mmol}$ ) in $100 \mu \mathrm{l}$ ASW containing $1 \%(\mathrm{wt} / \mathrm{vol})$ glucose and $0.1 \%(\mathrm{wt} / \mathrm{vol}) \mathrm{BSA}$. The ganglia were then washed free of unsequestered $\left[{ }^{3} \mathrm{H}\right] \mathrm{AA}$ prior to extraction for lipid. Points and error bars represent mean and SD of uptake into three ganglia. The ordinates depict uptake normalized to the amount of AA in the extracellular medium. (A) Uptake of AA into phospholipids. $(B)$ Uptake into triacylglycerol. (C) Uptake into the intracellular free AA pool

passing through an intracellular free pool. Therefore, constant release of free AA demonstrates that at least a portion of the internal free AA pool was constantly turning over.

\section{Uptake and Release of $A A$ from Sheath and Neurons}

After freezing in dry-ice cooled $80 \%$ ethylene glycol in ASW, labeled ganglia were dissected into neural and sheath components. The sheath was found to contain an average of $92 \%(n=6)$ of the radioactivity taken up by the whole ganglion. The sheath also contained about $95 \%$ of the whole ganglion lipid phosphate. Therefore, when normalized to phospholipid content, uptake of $\left.{ }^{3} \mathrm{H}\right] \mathrm{AA}$ into the sheath and neural components was about equal. Furthermore, we observed no differences between the two components in distribution of radioactivity in storage lipids, which was similar to the average distri-

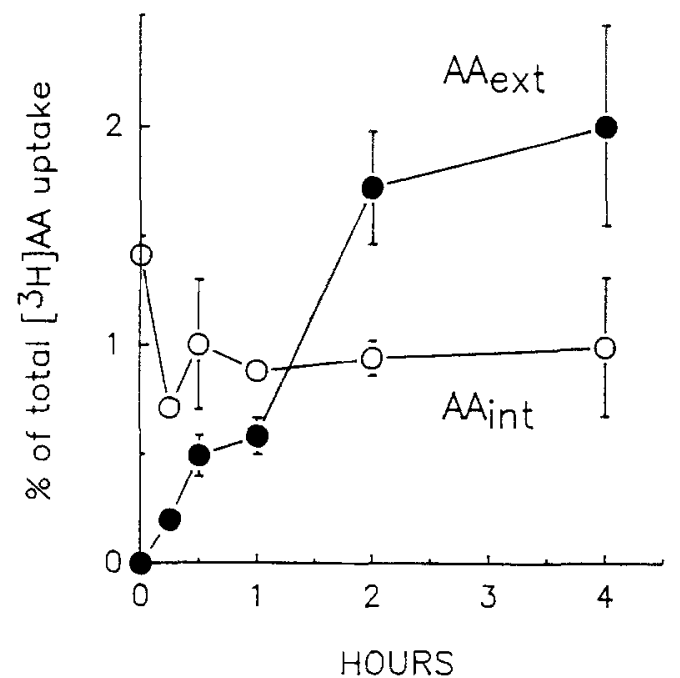

Fig. 3. Constant turnover of AA in Aplysia ganglia. Ganglia were labeled individually for $3 \mathrm{hr}$ in $150 \mu \mathrm{l}$ of $190 \mathrm{nM}\left[{ }^{3} \mathrm{H}\right] \mathrm{AA}$ (sp act: $240 \mathrm{Ci} / \mathrm{mmol}$ ). The ganglia were washed in fresh ASW and transferred into release incubation conditions, as described in Materials and Methods. Ganglia were removed from extracellular medium at the times indicated, and media and ganglia were extracted for lipid. Points and error bars represent mean and range of extracellular and intracellular free AA radioactivity from two ganglia, expressed as percentage of the total $\left[{ }^{3} \mathrm{H}\right] \mathrm{AA}$ originally taken up

bution for whole ganglia listed in the Table. However, the neural component was found to release $\mathrm{AA}$ and eicosanoids at nearly 10 times the rate of release from the sheath. Sheath and neural components were dissected apart in fresh, labeled ganglia, and the components were incubated separately in release incubation medium. After $15 \mathrm{~min}$, the medium of the neural component contained AA and eicosanoids at 7.0 and $10.6 \%$ (respectively) of the total radioactivity of neural component lipid. Over the same time period, the incubation medium of the sheath contained only 0.9 and $1.3 \%$ of the total radioactivity of sheath lipid in AA and eicosanoids.

\section{Pulse-Chase}

A pulse-chase protocol was used to further probe AA metabolism in Aplysia nervous system. Ganglia were "pulse"-labeled and then incubated with no exogenous AA (similar to Fig. 3) or with a chase of $50 \mu \mathrm{M}$ nonradioactive AA for 2 to $6 \mathrm{hr}$. Figure 4 shows the changes in relative content of radioactivity in individual lipid pools following incubation with this nonradioactive exogenous AA. Only phospholipid lost radioactivity in the presence of exogenous AA. The loss from PI was the greatest, and was the only substantial loss observed with both 2 


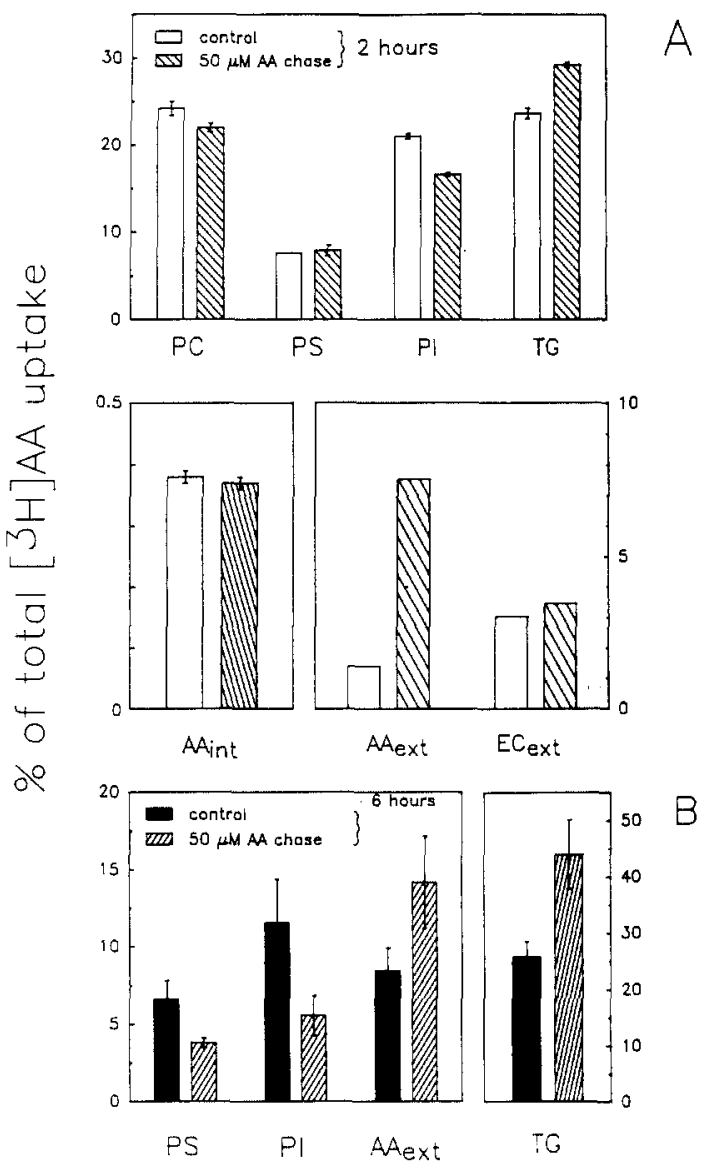

Fig. 4. Effect of nonradioactive exogenous AA on distribution of radioactivity. Ganglia were labeled for $3 \mathrm{hr}$ in 100-248 nM AA (sp act: 191-225 Ci/mmol), washed, and placed in medium containing no AA (equivalent to Fig. 3) or $50 \mu \mathrm{M}$ nonradioactive AA chase. Label in individual pools was determined after a 2 or $6 \mathrm{hr}$ incubation. Data in $A$ are from a 2 -hr incubation (open = control; right diagonal $=$ chase) . Radioactivity in major phospholipids, triacylglycerol, internal free AA, and free AA and eicosanoids released into medium is depicted. Bars and error bars are the mean and range of duplicate measurements. $\mathrm{AA}_{\mathrm{ext}}$ and $\mathrm{EC}_{\mathrm{ext}}$ are from a single measurement of two pooled samples. The decrease in radioactivity in PI and the increase in radioactivity in TG in the presence of exogenous AA were statistically significant at the $98 \%$ confidence level. Data in $B$ are from a 6 -hr incubation (solid $=$ control; left diagonal $=$ chase). Only pools in which radioactivity was significantly altered in the presence of exogenous AA are depicted. Bars and error bars are the mean and SD of triplicate measurements. Note for the bottom panels of $A$ and the panels of $B$ that the ordinate axes are on the left side of the left panels and the right side of the right panels

and $6 \mathrm{hr}$ incubations. PS also lost significant radioactivity by $6 \mathrm{hr}$; PC and PE did not show significant loss of radioactivity. The radioactivity lost from phospholipid appeared exclusively as increased radioactivity in two pools: triacylglycerol and free AA released into the extracellular medium. The radio-

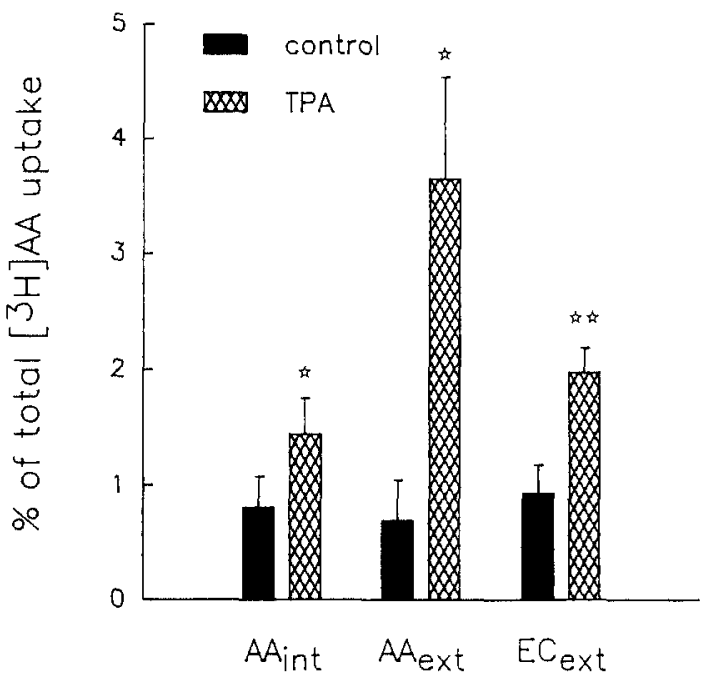

Fig. 5. TPA-induced increase in release of free AA and eicosanoids. Ganglia were labeled for $3 \mathrm{hr}$ in $150 \mu \mathrm{l}$ of $150 \mathrm{nM}\left[{ }^{3} \mathrm{H}\right] \mathrm{AA}$ (sp act: $240 \mathrm{Ci} / \mathrm{mmol}$ ) and prepared for assay of released products as described in Materials and Methods. The medium for the release incubation contained $500 \mathrm{nM}$ TPA (crosshatched) or carrier DMSO $(0.08 \%$, solid bars). After $60 \mathrm{~min}$ in release incubation, ganglia and media samples were removed for extraction of lipid. Bars and error bars are the mean and SD of triplicate measurements of radioactivity in internal free AA, secreted free AA, and eicosanoids. A star ( ative to control, at the $98 \%$ confidence level

activity in the internal free AA pool did not change. Also, radioactivity in eicosanoids released into the bathing medium was not altered in the presence of exogenous AA. Displacement of radioactive fatty acid from phospholipid to triacylglycerol or to the extracellular medium has been reported for other tissues incubated in the presence of exogenous fatty acid $[60,68,69]$.

\section{Modulation of Liberation of AA FROM PHOSPHOLIPID}

Veratridine or elevated $\mathrm{KCl}$ was used to depolarize ganglia. Labeled ganglia were exposed to $100 \mu \mathrm{M}$ veratridine for $15 \mathrm{~min}$ prior to the release incubation; for KCl-induced depolarization, ganglia were placed in $150-200 \mathrm{mM} \mathrm{KCl}$ at the start of the release incubation. Both depolarizing agents increased efflux of radioactivity into the extracellular medium up to twofold. Depolarization by elevated $\mathrm{KCl}$ was previously reported to stimulate release of eicosanoids from Aplysia ganglia [56]. The phorbol ester, TPA, also increased release of AA and eicosanoids from ganglia. In $500 \mathrm{nM}$ TPA, external release was stimulated on average fivefold, largely due to an increase in release of free AA (Fig. 5). Radioactivity 


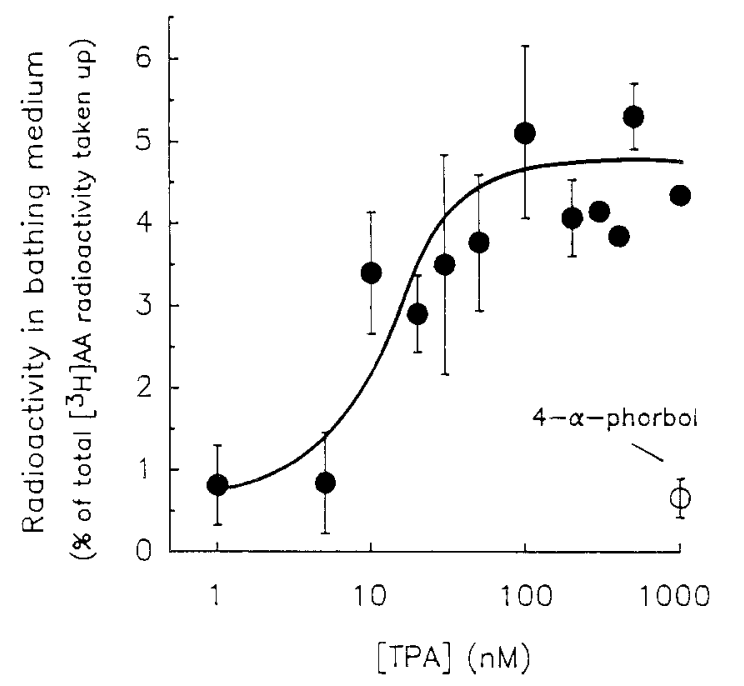

Fig. 6. Dose-response for TPA-stimulated release of radioactivity. Release of radioactivity was averaged from ganglia exposed to different concentrations of TPA. Labeling was for $2-3 \mathrm{hr}$ in $200-250 \mu \mathrm{l}$ of $8-18 \mathrm{nM}\left[{ }^{3} \mathrm{H}\right] \mathrm{AA}$ (sp act: $240 \mathrm{Ci} / \mathrm{mmol}$ ). Points with error bars represent mean and SD of release for $30 \mathrm{~min}$ from three to four ganglia. Points without error bars are single observations. The 4- $\alpha$-phorbol data point (open circle) was averaged from treatment of six ganglia with $1 \mu \mathrm{M} 4-\alpha$-phorbol. Radioactivity released was measured directly by removal and liquid scintillation counting of extracellular medium aliquots without further analysis

in internal free AA, and putative eicosanoids released into the medium, both increased about twofold (Fig. 5), in agreement with the general finding that the availability of intracellular AA is limiting for eicosanoid production [30].

The effect of TPA was dose dependent, with half-maximal stimulation at $15 \mathrm{~nm}$, and saturation above $100 \mathrm{~nm}$ (Fig. 6). This dose dependence is consistent with TPA acting through protein kinase $\mathrm{C}$ to stimulate liberation of AA from storage. Also, the lack of effect of the phorbol ester, 4- $\alpha$-phorbol (at $1 \mu \mathrm{M}$, see Fig. 6), which does not activate PKC in vitro, is consistent with involvement of $\mathrm{PKC}$ in the observed stimulation [12].

A loss of radioactivity from phospholipid, specifically PI and PC, accompanied the TPA-stimulated efflux of AA and eicosanoids into the extracellular medium. This change in distribution of radioactivity could have resulted from an inhibition of AA esterification into phospholipid, or stimulation of liberation from phospholipid. We tested the effect of TPA under the pulse-chase paradigm to distinguish between these possibilities. If TPA inhibited esterification into phospholipid, then TPA should have had less effect in the presence of a concentration of exogenous AA that saturated uptake into phospholipid (see Fig. 2). Figure 7 shows

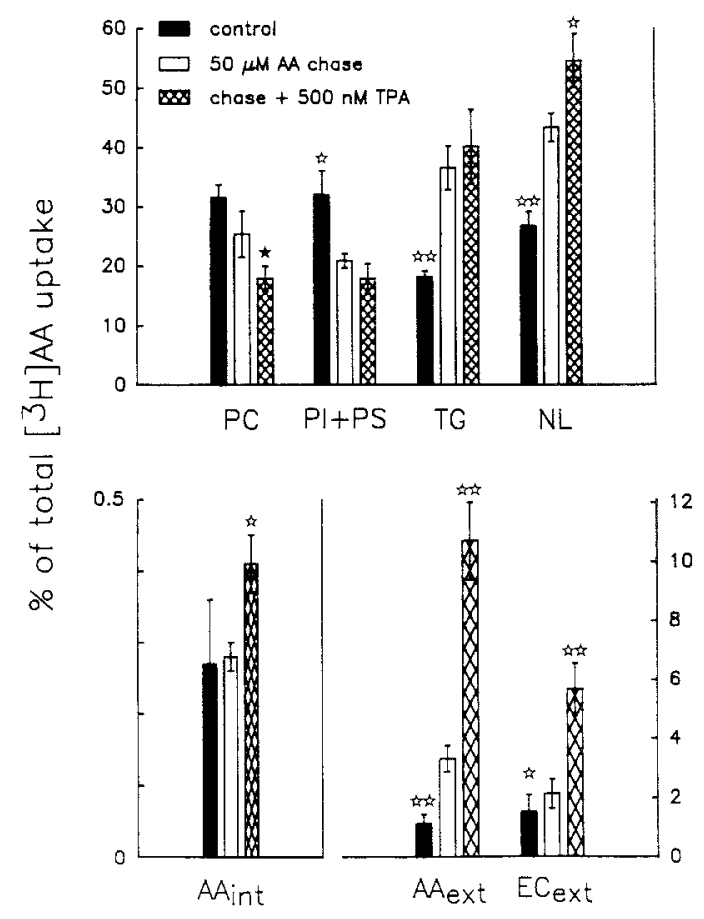

Fig. 7. Effect of TPA in the presence of exogenous AA. Ganglia were labeled for $3 \mathrm{hr}$ in $100 \mu \mathrm{l}$ of $115 \mathrm{nM}\left[{ }^{3} \mathrm{H}\right] \mathrm{AA}$ (sp act: $225 \mathrm{Ci}$ / $\mathrm{mmol}$ ), washed free of unsequestered $\mathrm{AA}$, and incubated for $6 \mathrm{hr}$ in release incubation medium alone (filled), with $50 \mu \mathrm{M}$ exogenous AA (open), or with $50 \mu \mathrm{M}$ AA plus $500 \mathrm{nM}$ TPA (crosshatched). The data from control and chase alone in this figure are comparable to the results of similar experiments depicted in Fig. 4. Extracellular medium was removed and replaced hourly. Ganglia and medium were extracted for lipid, and the extracts were analyzed as described in Materials and Methods. Bars and error bars are the mean and SD of triplicate measurements. Symbols above the bars indicate a significant difference relative to chase for the following confidence levels: filled star $=95 \%$; open star $=$ $98 \%$; two open stars $=99.5 \%$

TPA still had a profound effect under these conditions, increasing loss of radioactivity from phospholipid, efflux of radioactive free AA and eicosanoids, and accumulation of radioactivity in neutral lipid relative to chase alone. Also, the internal free AA radioactivity increased nearly twofold, with a commensurate increase in radioactivity in eicosanoids.

The effects of both TPA and depolarizing agents were strictly dependent on extracellular calcium, suggesting at least a partially shared mechanism of activation. Substitution of calcium in the extracellular medium with $20 \mathrm{~mm}$ cobalt, a blocker of voltage-dependent calcium channels, completely suppressed the response to depolarizing agents or TPA. Also, lowering calcium in the medium from 11 $\mathrm{mM}$ to $100 \mu \mathrm{M}$ was sufficient to block the effect of TPA. Raising extracellular calcium to $60 \mathrm{~mm}$ increased TPA-induced stimulation about twofold. Although lowering calcium in the medium did not 


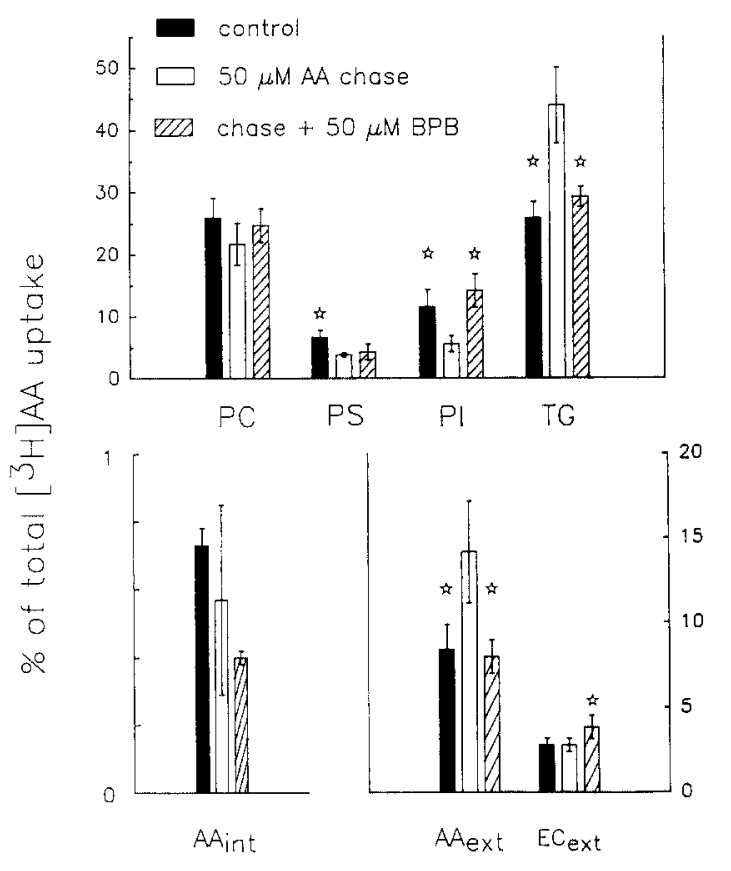

Fig. 8. Effect of BPB in the presence of high exogenous AA. Ganglia were labeled individually for $3 \mathrm{hr}$ in $100 \mu \mathrm{l}$ of $108 \mathrm{nM}$ [ $\left.{ }^{3} \mathrm{H}\right] \mathrm{AA}$ (sp act: $191 \mathrm{Ci} / \mathrm{mmol}$ ), washed, and incubated for $6 \mathrm{hr}$ in release incubation medium alone (filled), or with $50 \mu \mathrm{M}$ AA (open), or with $50 \mu \mathrm{M} \mathrm{AA}+50 \mu \mathrm{M}$ BPB (left diagonal). The control and chase data in this figure are comparable to the results of similar experiments depicted in Figs. 4 and 7. The extracellular medium was removed and replaced hourly. Ganglia and medium were extracted for lipid and the extracts were analyzed as described in Materials and Methods. Bars and error bars are the mean SD of quadruplicate measurements. An open star (t) indicates significance difference, relative to chase, at the $98 \%$ confidence level

affect basal release of radioactivity, raising bath calcium to $50 \mathrm{~mm}$ increased basal release from $4.3 \%$ of uptake/hr to $10.5 \% / \mathrm{hr}$.

4-bromophenacylbromide is established as an irreversible inactivator of phospholipase $\mathrm{A}_{2}[58$, 71]. Figure 8 depicts the effect of $50 \mu \mathrm{M}$ BPB in the presence of $50 \mu \mathrm{M}$ exogenous AA chase. The chase-induced loss of radioactivity from PI and increase in radioactivity in triacylglycerol and free $\mathrm{AA}$ in the medium were completely inhibited in the presence of $\mathrm{BPB}$. Also, in the presence of exogenous $\mathrm{AA}$ and $\mathrm{BPB}$, radioactivity in internal free $\mathrm{AA}$ was $48 \pm 13 \%(n=7)$ less than in exogenous AA alone. These results are consistent with inhibition of phospholipase by BPB.

\section{Discussion}

\section{Free AA Buffering}

Uptake of radioactivity into the internal free AA pool did not saturate in up to $25 \mu \mathrm{M}$ exogenous AA.
This demonstrated a large flux of exogenous AA into the internal AA pool was occurring in the presence of $25 \mu \mathrm{M} \mathrm{AA}$. If the size of the internal free pool was significantly increased due to this influx of exogenous AA, the resulting decrease in the specific activity should have been detected as a decrease in radioactivity in AA and eicosanoids released into the extracellular medium. The substantial increase in release of radioactive free $\mathrm{AA}$, and lack of change (or a very slight increase) in release of radioactive eicosanoids during the chase, suggests $50 \mu \mathrm{M}$ exogenous AA did not significantly change the size of the internal free AA pool or the resting level of eicosanoid production. In other words, the AA that is taken up must be converted immediately to some other form, likely AA-CoA, which makes the AA unavailable for conversion to eicosanoids. This result is critical, because it suggests application of exogenous AA may not be an effective means of increasing eicosanoid production for investigation of eicosanoid-mediated physiology. If exogenous AA does not change the internal free AA pool size or eicosanoid production, acute physiological effects observed with the use of exogenous AA cannot be a result of increased eicosanoid production, but most likely result from direct actions of AA, acting at external receptors, or from altered AA content in glycerolipids. We plan to test this interpretation of our pulse-chase data by gasliquid chromatographic measurement of free AA concentration and radioimmunoassay for specific eicosanoids.

If this interpretation is correct, pathways responsible for esterification of AA into lipid must buffer internal AA efficiently enough to prevent a significant increase in internal free AA in the presence of high external AA. Acyl-CoA synthetase activity (Fig. 1, pathway $I I$ ) is a good candidate for rapid sequestration of free $\mathrm{AA}$ in the presence of an exogenous source. An acyl-CoA synthetase which favors 20 carbon unsaturated fatty acids as substrates was isolated from platelets in an effort to understand how the level of free AA is maintained so low relative to other fatty acids $[49,73]$. This arachidonyl-CoA synthetase exhibited nanomolar affinity for AA, the highest affinity among the fatty acids tested, and had a very high turnover rate. Since this first demonstration, arachidonyl-CoA synthetase activity has been measured in many tissues [36], and, although untested, Aplysia nervous system may also contain this activity.

If the size of the internal free AA pool does not change significantly in the presence of a high concentration of exogenous AA, how can we explain the different characteristics of uptake into storage lipids as a function of $A A$ concentration? If synthetase activity rapidly converts AA to AA-CoA, the 
AA-CoA concentration likely increases with increasing exogenous AA. Intracellular accumulation of exogenous long chain fatty acids as CoA-esters has been shown to inhibit $\beta$-oxidation of other fatty acids, due to sequestration of acetyl-CoA $[3,53]$. If the size of the AA-CoA pool increases with increasing exogenous $\mathrm{AA}$, the results of the uptake experiments could be interpreted to reflect the different affinities of fatty acyl-CoA transferases for AACoA. Transferases for esterification into phospholipid may have high affinity for AA-CoA, but saturate at a relatively low concentration; the diglyceride acyltransferases may have somewhat lower affinity, but may not reach saturation for the concentration of AA-CoA produced in up to $50 \mu \mathrm{M}$ AA. The combination of a high affinity synthetase with rapid turnover, and two transferases with affinities for AA-CoA covering a broad range of AA-CoA concentration, could provide an efficient buffering system for free AA.

\section{Phospholipase Activity}

The phospholipase activity responsible for liberation of AA from phospholipid could involve $\mathrm{PLA}_{2}$ or phospholipase $\mathrm{C}$. Direct hydrolytic removal of AA by $\mathrm{PLA}_{2}$, or indirect liberation through the activity of PLC on phosphoinositides to produce diacylglycerol which is broken down into monoacylglycerol and AA, are the primary mechanisms for liberation of AA from phospholipid. Which of these is the most important remains in dispute [30, $34,61]$. $\mathrm{BPB}$ is an irreversible inhibitor of purified $\mathrm{PLA}_{2}$ in vitro [58, 71], and $\mathrm{BPB}$ has been shown to inhibit $\mathrm{PLA}_{2}$ (as indicated by inhibition of AA release from phospholipid) in vivo, in a variety of tissues $[1,8,29,31,65]$, and in an irreversible manner [9, 42]. In Aplysia ganglia, BPB inhibited the effect of chase, and decreased internal free AA, suggesting involvement of $\mathrm{PLA}_{2}$ in basal liberation of AA from phospholipid. However, this effect of $\mathrm{BPB}$ is not sufficient to conclude PLA $\mathrm{P}_{2}$ is the most important pathway for liberation of AA in Aplysia.

The calcium dependence of TPA- or depolarization-induced stimulation of AA liberation suggests the involvements of PLA $A_{2}$. Purified PLA $\mathrm{PL}_{2}$ is strictly dependent on added calcium, and several isoforms purified from sheep platelets respond to changes in free calcium in the micromolar range [38], suggesting the potential for calcium-mediated activation of PLA $_{2}$ within a physiological range of calcium concentrations. In contrast, PLC functions optimally with resting levels of free calcium; therefore, increasing intracellular calcium generally does not activate PLC $[6,44]$.

The specific phospholipid source of AA varies between tissues. PC and PE are commonly in- volved; however, specific loss of AA from PI has been reported. PI is the primary source of stimulated AA liberation in platelets [73]. In a glioma cell line, acetylcholine stimulated specific loss of AA from PI in a mechanism involving initial activation

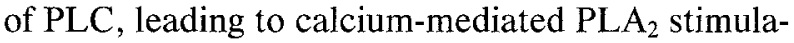
tion and breakdown of PI to AA and lysophosphatidylinositol [14]. Parallel stimulation of PLC and $\mathrm{PLA}_{2}$ in canine kidney cells has been shown to occur with $\alpha_{1}$-adrenoceptor activation, leading to liberation of AA from PI [66]. Specific PLA -catalyzed $_{2}$ liberation of AA from PI has also been demonstrated in mouse macrophages $[20,21]$. The latter studies are among many examples of phorbol esterstimulated liberation of AA from phospholipid, believed to result from $\mathrm{PLA}_{2}$ activation $[24,26,27$, 51].

Both BPB and TPA affected AA metabolism in Aplysia, likely through modulation of phospholipase activity. TPA was tested in the pulse-chase experiments to determine if TPA-stimulated AA turnover was due to inactivation of phospholipase or inhibition of enzymes catalyzing esterification. TPA was still very effective with chase; therefore, TPA must stimulate phospholipase activity in Aplysia. This is consistent with the possibility phospholipase activity is rate limiting for turnover of AA, as has been observed in other systems [28, 30]. If phospholipase activity is not at least partially rate limiting, activation or inhibition of this activity (by TPA and BPB, respectively) would not have had such a pronounced effect. Also, in a preliminary experiment, we found BPB inhibited uptake of AA into phospholipid about 50\% (data not shown). Therefore, we conclude phospholipase activity is probably rate limiting for turnover of free $\mathrm{AA}$ in Aplysia ganglia.

\section{Protein Kinase C}

The dose dependence of TPA stimulation suggests participation of protein kinase $C$ in the phospholipase activation mechanism. However, we do not know whether protein kinase $\mathrm{C}$ directly activates phospholipase through phosphorylation of the enzyme. Phorbol esters have been shown to increase voltage-dependent calcium currents in two different neuronal cell types in Aplysia, the bag cell neurons of the abdominal ganglion [17], and the sensory neurons of the pleural ganglion [7]; this might occur in other neurons as well. Therefore, TPA-mediated activation of protein kinase $C$ could cause an increase in intracellular calcium which would then stimulate phospholipase activity. A similar mechanism may account for depolarization-induced release of AA and eicosanoids. However, in vitro depolarization is an inefficient means for loading cells 
with calcium, because a sustained increase in intracellular calcium will rapidly inactivate calcium channels [19]. Depolarization may prove a more potent stimulator of AA turnover if studied with electrically-induced depolarizations of physiological duration.

\section{Uptake and Metabolism by Sheath}

Although almost all of the radioactivity taken up into whole ganglia was sequestered by the sheath, the use of whole ganglia in this study does not detract from the results. First, the ganglionic sheath of Aplysia and many other molluscs is known to contain many neuritic processes $[4,5,18,37,59,62]$. The similarity between the rate of AA uptake and distribution of radioactivity in storage lipids, and distribution of radioactivity in released AA and eicosanoids in neural and sheath components, suggest the neuritic processes present in the sheath may be largely responsible for the observed AA metabolism. Second, we have found that exogenous AA is sequestered avidly by isolated clusters of cell bodies and Aplysia neurons in primary culture, and we also found that TPA stimulated release of radioactivity from the labeled clusters of cell bodies (data not shown). Third, the prodigious rate of release of AA and eicosanoids from the isolated neural component suggests the magnitude of the results reported in this paper would only be larger if this work were repeated with isolated cell bodies. Finally, the regulation of AA metabolism is known to involve intercellular, or paracrine interactions, as has been shown for interactions between platelets, white blood cells, and endothelium $[35,39,40,41$, 64]. Therefore, the study of AA metabolism on the level of the whole ganglia is most meaningful for eventual in vivo extrapolation of these results.

\section{SECRETION OF AA AND EICOSANOIDS}

A substantial rate of intracellular AA turnover has been observed in many types of cells in culture [67]. Also, constant release of radioactive free fatty acids with lack of concomitant change in internal free fatty acid radioactivity has been observed in bovine aortic endothelial cells in culture [22]. However, we are not aware of a report of constant release of eicosanoids at rest. Based upon the finding that depolarization stimulated eicosanoid production in Aplysia, it is possible spontaneous neural activity may account for this "resting" eicosanoid production. The total release of radioactive free AA and eicosanoids averaged $\sim 2 \%$ of total uptake per hour of release incubation. If futile, this constant loss of AA by secretion or conversion to eicosanoids must be expensive for the cell to maintain. Also, eicosanoids have multiple biological activities and are typically active at very low concentrations. A random, futile production of eicosanoids would likely have undesirable physiological effects. We conclude that this considerable and constant net loss of AA and eicosanoids may not be the result of futile cycling, but rather it is more likely the by-product of a rapid deacylation-reacylation cycle which regulates intracellular free AA tightly. In the accompanying paper [10], we investigate the possibility that this constant cycling or turnover has a role in regulation of electrical activity in Aplysia nervous system.

This work was supported by NIH grant NS17910 to I. B. L. We are grateful to David Wilson for his critical comments on the manuscript.

\section{References}

1. Abdel-Latif, A.A., Smith, J.P. 1982. Studies on the incorporation of $\left[1-{ }^{14} \mathrm{C}\right]$ arachidonic acid into glycerolipids and its conversion into prostaglandins by rabbit iris. Effects of antiinflammatory drugs and phospholipase $\mathrm{A}_{2}$ inhibitors. Biochim. Biophys. Acta 711:478-489

2. Aguilera, G., Abou-Samra, A.B., Harwood, J.P., Catt, K.J. 1988. Corticotropin-releasing factor receptors: Characterization and actions in the anterior pituitary. Adv. Exp. Med. Biol. 245:83-105

3. Bar-Tana, J., Rose-Kahn, G., Srebnik, M. 1985. Inhibition of lipid synthesis by $\beta \beta^{\prime}$-tetramethylated-substituted, $\mathrm{C}_{14^{-}}$ $\mathrm{C}_{22}, \alpha, \omega$-dicarboxylic acids in the rat in vivo. J. Biol. Chem. 260:8404-8410

4. Benjamin, P. R., Peat, A. 1968. Myoneural junctions in the connective tissue sheath of a molluscan ganglion. Nature (London) 219:1371-1372

5. Benjamin, P.R., Peat, A. 1971. On the structure of the pulmonate osphradium. II. Ultrastructure. Z. Zellforsch. Mikrosk. Anat. 118:168-189

6. Billah, M.M., Lapetina, E.G. 1982. Degradation of phosphatidylinositol-4,5-bisphosphate is insensitive to $\mathrm{Ca}^{2+}$ mobilization in stimulated platelets. Biochem. Biophys. Res. Commun. 109:217-222

7. Braha, O., Klein, M., Kandel, E.R. 1988. Phorbol ester increases $\mathrm{Ca}^{++}$current in Aplysia sensory neurons. Soc. Neurosci. Abstr. 17:644

8. Camorrato, A.M., Grandison, L. 1985. Evidence supporting a correlation between arachidonic acid release and prolactin secretion from $\mathrm{GH}_{3}$ cells. Endocrinology 116:1506-1513

9. Carine, K., Hudig, D. 1984. Assessment of a role for phospholipase $\mathrm{A}_{2}$ and arachidonic acid metabolism in human lymphocyte natural cytotoxicity. Cell. Immunol. 87:270-283

10. Carlson, R.O., Levitan, I.B. 1990. Constant turnover of free AA and constitutive inhibition of a potassium current in Aplysia giant neurons. J. Membrane Biol. 116:261-272

11. Cashman, J.R. 1989. 5,6-Epoxyeicosatrienoic acid stimulates growth hormone release in rat anterior pituitary cells. Life Sci 44: 1387-1393

12. Castagna, M., Takai, Y., Kaibuchi, K., Sano, K., Kikkawa, U., Nishizuka, Y. 1982. Direct activation of calcium-acti- 
vated, phospholipid-dependent protein kinase by tumor-promoting phorbol esters. J. Biol. Chem. 257:7847-7851

13. Davies, P. 1984. The role of oxygenation products in pain and inflammation. Annu. Rev. Immunol. 2:335-357

14. DeGeorge, J.J., Ousley, A.H., McCarthy, K.D., Lapetina, E.G., Morell, P. 1987. Acetylcholine stimulates selective liberation and re-esterification of arachidonate and accumulation of inositol phosphates and glycero-phosphoinositol in C62B glioma cells. J. Biol. Chem. 262:8077-8083

15. DeGrella, R.F., Light, R.J. 1980. Uptake and metabolism of fatty acids by dispersed adult rat heart myocytes: Kinetics of homologous fatty acids. J. Biol. Chem. 255:9731-9738

16. DeGrella, R.F., Light, R.J, 1980. Uptake and metabolism of fatty acids by dispersed adult rat heart myocytes: Inhibition by albumin and fatty acid homologues. J. Biol. Chem. 255:9739-9745

17. DeRiemer, S.A., Strong, J.A., Albert, K.A., Greengard, P., Kaczmarek, L.K. 1985. Enhancement of calcium current in Aplysia neurons by phorbol ester and protein kinase $\mathrm{C}$. $\mathrm{Na}$ ture (London) 313:313-316

18. Dyer, R.F., Cowden, R.R. 1973. Electron microscopy of the esophogeal ganglion complex of the gastropod pulmonate Triodopsis divesta: I. Ultrastructure of the epineurium. $J$. Morphol. 139:125-153

19. Eckert, R., Tillotson, D.L. 1981. Calcium-mediated inactivation of the calcium conductance in caesium-loaded giant neurones of Aplysia californica. J. Physiol. (London) 314:265-280

20. Emilsson, A., Sundler, R. 1986. Evidence for a catalytic role of phospholipase $\mathrm{A}$ in phorbol diester- and zymosan-induced mobilization of arachidonic acid in mouse peritoneal macrophages. Biochim. Biophys, Acta 876:533-542

21. Emilsson, A., Wijkander, J., Sundler, R. 1986. Diacylglycerol induces deacylation of phosphatidylinositol and mobilization of arachidonic acid in mouse macrophages. Comparison with induction by phorbol diester. Biochem. $J$. 239:685-690

22. Figard, P.H., Hejlik, D.P., Kaduce, T.L., Stoll, L.L., Spector, A.A. 1986. Free fatty acid release from endothelial cells. J. Lipid Res. 27:771-780

23. Folch, J., Lees, M., Sloane-Stanley, G.H. 1957. A simple method for the isolation and purification of total lipides from animal tissues. J. Biol. Chem. 226:497-509

24. Frye, R.A., Holz, R.W. 1985. Arachidonic acid release and catecholamine secretion from digitonin-treated chromaffin cells: Effects of micromolar calcium, phorbol ester, and protein alkylating agents. J. Neurchem. 44:265-273

25. Heape, A.M., Juguelin, H., Boiron, F., Cassagne, C. 1985. Improved one-dimensional thin-layer chromatographic technique for polar lipids. J. Chromatogr. 322:391-395

26. Hirata, F., Corcoran, K., Venkatasubramanian, K., Schiffman, E., Axelrod, J. 1979. Chemoattractants stimulate degradation of methylated phospholipid and release of arachidonic acid in rabbit leukocytes. Proc. Natl. Acad. Sci. USA 76:2640-2643

27. Ho, A.K., Klein, D.C. 1987. Activation for alpha1-adrenoceptors, protein kinase $C$, or treatment with intracellular free $\mathrm{Ca}^{2+}$ elevating agents increases pineal phospholipase $\mathrm{A}_{2}$ activity. J. Biol. Chem. 262:11764-11770

28. Hong, S.L. 1988. The release of arachidonic acid from cellular lipids. Prog. Allergy 44:99-139

29. Ida, E., Sakata, A., Tominaga, M., Yamasaki, H., Onoue, K. 1988. Arachidonic acid release is closely related to the Fe gamma receptor-mediated superoxide generation in macrophages. Microbiol. Immunol. 32:1127-1143
30. Irvine, R.F. 1982. How is the level of free arachidonic acid controlled in mammalian cells? Biochem. J. 204:3-16

31. Johns, R.A., Izzo, N.J., Milner, P.J., Loeb, A.L., Peach, M.J. 1988. Use of cultured cells to study the relationship between arachidonic acid and endothelium-derived relaxing factor. Am. J. Med. Sci. 295:287-292

32. Judd, A.M., Spangelo, B.L., Ehreth, J.T., MacLeod, R.M. 1988. A possible role for lipoxygenase and epoxygenase arachidonate metabolites in prolactin release from pituitary cells. Neuroendocrinology 48:407-416

33. Keenan, R.W., Schmidt, G., Tanaka, T. 1968. Quantitative determination of phosphatidal ethanolamine and other phosphatides in various tissues of the rat. Anal. Biochem. 23:555-566

34. Lagarde, M. 1988. Metabolism of fatty acids by platelets and the functions of various metabolites in mediating platelet function. Prog. Lipid Res. 27:135-152

35. Lagarde, M., Gualde, N., Rigaud, M. 1989. Metabolic interactions between eicosanoids in blood and vascular cells. Biochem. J. 257:313-320

36. Laposata, M., Reich, E.L., Majerus, P.W. 1985. Arachidonoyl-CoA synthetase. Separation from nonspecific acyl-CoA synthetase and distribution in various cells and tissues. $J$. Biol. Chem. 260:11016-11020

37. Lieberman, A.R. 1968. The connective tissue elements of the mammalian nodose ganglion. An electron microscope study. Z. Zellforsch. Mikrosk. Anat. 89:95-111

38. Loeb, L.A., Gross, R.W. 1986. Identification and purification of sheep platelet phospholipase $\mathrm{A}_{2}$ isoforms. Activation by physiologic concentrations of calcium ion. J. Biol. Chem. 261: $10467-10470$

39. Maclouf, J., Del, M.A., Grange, M.J., Corvazier, E., Borgeat, P. 1985. Regulation and manipulation of arachidonate cascade in cell-cell interaction. Adv. Prostaglandin Thromboxane Leukotriene Res. 15:209-211

40. Maclouf, J.A., Murphy, R.C. 1988. Transcellular metabolism of neutrophil-derived leukotriene $\mathrm{A}_{4}$ by human platelets. A potential cellular source of leukotriene $\mathrm{C}_{4} . \mathrm{J} . \mathrm{Biol}$. Chem. 263:174-181

41. Marcus, A.J. 1986. The role of thrombin in transcellular metabolism of eicosanoids. Ann. NY Acad. Sci. 485:369-373

42. Marone, G., Fimiani, B., Torella, G., Poto, S., Bianco, P., Condorelli, M. 1983. Possible role of arachidonic acid and of phospholipase $\mathrm{A}_{2}$ in the control of lysosomal enzyme release from human polymorphonuclear leukocytes. J. Clin. Lab. Immunol. 12:111-116

43. Metz, S.A. 1988. Arachidonic acid and its metabolites: Evolving roles as transmembrane signals for insulin release. Prostaglandins Leukot. Essent. Fatty Acids. 32:187-202

44. Michell, R.H., Kirk, C.J., Jones, L.M., Downes, C.P., Creba, J.A. 1981. The stimulation inositol lipid metabolism that accompanies calcium mobilization in stimulated cells: Defined characteristics and unanswered questions. Phil. Trans. R. Soc. London 296:123-138

45. Miyake, A., Nishizaki, T., Ikegami, H., Koike, K., Hirota, K., Tanizawa, O. 1988. Possible involvement of lipoxygenase pathway of arachidonic acid in rat pituitary hormone release in vitro. J. Endocrinol. Invest. 11:805-808

46. Myher, J.J. 1978. Separation and determination of the structure of acylglycerols and their ether analogues. In: Handbook of Lipid Research. A.A. Kuksis, editor. Plenum, New York-London

47. Needleman, P., Turk, J., Jakschik, B.A., Morrison, A.R., Lefkowith, J.B. 1986. Arachidonic acid metabolism. Annu. Rev. Biochem. 55:69-102 
48. Negro-Vilar, A., Conte, D., Valenca, M. 1986. Transmembrane signals mediating neural peptide secretion: Role of protein kinase $\mathrm{C}$ activators and arachidonic acid metabolites in luteinizing hormone-releasing hormone secretion. Endocrinology 119:2796-2802

49. Neufeld, E.J., Wilson, D.B., Sprecher, H., Majerus, P.W. 1983. High affinity esterification of eicosanoid precursor fatty acids by platelets. J. Clin. Invest. 72:214-220

50. Nishizaki, T., Ikegami, H., Tasaka, K., Hirota, K., Miyake, A., Tanizawa, O. 1989. Mechanism of release of beta-endorphin from rat pituitary cells. Role of lipoxygenase products of arachidonic acid. Neuroendocrinology 49:483-488

51. Ohuchi, K., Levine, L. 1978. Stimulation of prostaglandin synthesis by tumor-promoting phorbol-12, 13-diesters in canine kidney (MDCK) cells. Cycloheximide inhibits the stimulated prostaglandin synthesis, deacylation of lipids, and morphological changes. J. Biol. Chem. 253:4783-4790

52. Ojeda, S.R., Capdevila, J., Snyder, G., McCann, S.M., Negro Vilar, A., Falck, J.R. 1985. Involvement of arachidonic acid metabolites in the control of hypothalamic-pituitary function. Adv. Prostaglandin Thromboxane Leukotriene Res. 15:559-560

53. Osmundsen, H., Bjornstad, K. 1985. Inhibitory effect of some long-chain unsaturated fatty acids on mitochondrial beta-oxidation. Effects of streptozotocin-induced diabetes on mitochondrial beta-oxidation of polyunsaturated fatty acids. Biochem. I. 230:329-337

54. Parker, C.W. 1987. Lipid mediators produced through the lipoxygenase pathway. Annu. Rev. Immunol. 5:65-84

55. Piomelli, D., Shapiro, E., Feinmark, S.J., Schwartz, J.H. 1987. Metabolites of arachidonic acid in the nervous system of Aplysia: Possible mediators of synaptic modulation. $J$. Neurosci. 7:3675-3686

56. Piomelli, D., Volterra, A., Dale, N., Siegelbaum, S.A., Kandel, E.R., Schwartz, J.H., Belardetti, F. 1987. Lipoxygenase metabolites of arachidonic acid as second messengers for presynaptic inhibition of Aplysia sensory cells. Nature (London) 328: 38-43

57. Prentki, M., Matschinsky, F.M. 1987. $\mathrm{Ca}^{2+}$, cAMP, and phospholipid-derived messengers in coupling mechanisms of insulin secretion. Physiol. Rev. 67:1185-1248

58. Roberts, M.F., Deems, R.A., Mincey, T.C., Dennis, E.A. 1977. Chemical modification of the histidine residue in phospholipase $\mathrm{A}_{2}$ (Naja naja naja). J. Biol. Chem. 252:2405-2411

59. Rogers, D.C. 1969. Fine structure of the epineural connective tissue sheath of the subesophageal ganglion in Helix. aspersa. Z. Zellforch. Mikrosk. Anat. 102:99-112

60. Rosenthal, M.C., Geyer, R.P. 1977. Release of phospholipid acyl groups from fetal human fibroblasts. Biochem. Biophys. Res. Commun. 74:1456-1462
61. Rosenthal, M.D. 1987. Fatty acid metabolism of isolated mammalian cells. Prog. Lipid Res. 26:87-124

62. Sanchis, C.A., Zambrano, D. 1969. The structure of the central nervous system of a pulmonate mollusc (Cryptomphallus aspersa): I. Ultrastructure of the connective epidural sheath. Z. Zellforsch. Mikrosk. Anat. 94:62-71

63. Sanui, H. 1974. Measurement of inorganic orthophosphate in biological materials: Extraction properties of butyl acetate. Anal. Biochem. 60:489-504

64. Schafer, A. I., Crawford, D.D., Gimbrone, M.J. 1984. Unidirectional transfer of prostaglandin endoperoxides between platelets and endothelial cells. J. Clin. Invest. 73:1105-1112

65. Shirazi, Y., McMorris, F.A., Shin, M.L. 1989. Arachidonic acid mobilization and phosphoinositide turnover by the terminal complement complex, C5b-9, in rat oligodendrocyte $x$ C6 glioma cell hybrids. J. Immunol. 142:4385-4391

66. Slivka, S.R., Insel, P.A. 1987. Alpha 1-adrenergic receptormediated phosphoinositide hydrolysis and prostaglandin $\mathrm{E}_{2}$ formation in Madin-Darby canine kidney cells. Possible parallel activation of phospholipase $\mathrm{C}$ and phospholipase $\mathrm{A}_{2} . J$. Biol. Chem. 262:4200-4207

67. Spector, A.A., Mathur, S.N., Kaduce, T.L., Hyman, B.T. 1980. Lipid nutrition and metabolism of cultured mammalian cells. Prog. Lipid Res. 19:155-86

68. Tsai, P.Y., Geyer, R.P. 1977. Fatty acid synthesis and metabolism of phospholipid acyl groups in strain L mouse fibroblasts. Biochim. Biophys. Acta 489:381-389

69. Tsai, P.Y., Geyer, R.P. 1978. Effect of exogenous fatty acids on the retention of phospholipid acyl groups by mouse L fibroblasts. Biochim. Biophys. Acta 528:344-354

70. Turk, J., Wolf, B.A., McDaniel, M.L. 1987. The role of phospholipid-derived mediators including arachidonic acid, its metabolites, and inositol-trisphosphate and of intracellular $\mathrm{Ca}^{2+}$ in glucose-induced insulin secretion by pancreatic islets. Prog. Lipid Res. 26:125-181

71. Volwerk, J.J., Pieterson, W.A., de Haas, G.H. 1974. Histidine at the active site of phospholipase $\mathrm{A}_{2}$. Biochemistry 13:1446-1454

72. Williams, K.I., Higgs, G.A. 1988. Eicosanoids and inflammation. J. Pathol, 156:101-110

73. Wilson, D.B., Prescott, S.M., Majerus, P.W. 1982. Discovery of an arachidonoyl coenzyme-A synthetase in human platelets. J. Biol. Chem. 257:3510-3515

74. Wolf, B.A., Colca, J.R., Turk, J., Florholmen, J., McDaniel, M.L. 1988, Regulation of $\mathrm{Ca}^{2+}$ homeostasis by islet endoplasmic reticulum and its role in insulin secretion. Am. J. Physiol. 254:E121-E136

Received 27 October 1989 